Modeling, Identification and Control, Vol. 33, No. 2, 2012, pp. 35-44, ISSN 1890-1328

\title{
Discrete LQ optimal control with integral action: A simple controller on incremental form for MIMO systems
}

\author{
David Di Ruscio
}

Telemark University College, Faculty of Technology, N-3918 Porsgrunn, Norway. Fax: +4735 57 54 01, Tel: +47 355751 68, E-mail: David.Di.Ruscio@hit.no

\begin{abstract}
A simple Linear Quadratic (LQ) optimal controller of velocity (incremental) form with approximately the same properties as a conventional PID controller of velocity form is presented, i.e. integral action. The proposed optimal controller is insensitive to slowly varying system and measurement trends and has the ability of stabilizing any linear dynamic system under weak assumptions such as the stabilizability of the system and the detectability of the system seen from the performance index.
\end{abstract}

Keywords: MIMO systems, optimal controller, integral action, PI controller, Kalman filter, system identification

\section{Introduction}

The famous Linear Quadratic (LQ) optimal controller for linear Multiple Input and Multiple Output (MIMO) systems (se. e.g. Kwakernaak and Sivan (1972) and Anderson and Moore (1989)), has some remarkable properties due to the guaranteed nominal stability of the closed loop controlled system (under weak conditions such as the stabilizability of the system and the detectability of the system seen from the objective).

On the other hand, this LQ optimal controller has not attained the position it deserves. One reason for this is probably that it has been difficult to compare the LQ optimal controller with a standard PID controller which has received a great deal of attention owing to its simplicity and its practical applications.

In this paper we will show how we may use the standard LQ optimal controller to design stabilizing controllers for MIMO systems with approximately the same properties that a PI controller has on velocity (incremental) form.

The proposed controller is remarkably simple and it has almost the same structure and properties as a standard PID controller, i.e., the controller has integral action.

The proposed controller also has the properties of stabilizing any detectable and stabilizable linear MIMO system. Hence, the resulting controller may be used as a first choice controller for controlling a linear system.

The main contributions of this paper are itemized as follows:

- A LQ optimal controller for discrete time systems with approximately the same properties as a standard PID controller is proposed.

- The proposed LQ optimal controller may be used for finding stabilizing controllers with integral action for complex MIMO systems.

- The proposed LQ optimal controller is insensitive to constant or slowly varying process and measurement noise.

- The proposed controller is suitable for controlling 
non-linear systems when a linear state space model is available. This linear model may be the result of linearizing a non-linear model or the result of system identification.

- The proposed LQ optimal controller is illustrated on four non-linear process models, e.g. the quadruple tank process Johansson (2002).

- The proposed LQ optimal controller is in this paper also illustrated with practical experiments on a quadruple tank laboratory process. System identification is used to identify a state space model on innovations form (The Kalman filter) and used in the design of the proposed LQ controller. This strategy may be viewed as a "model free" LQ optimal controller strategy because only process data are used.

The main differences of the proposed MIMO PI LQ optimal controller and a conventional PI controller is that the optimal controller consists of both output and state feedback, while a conventional PI controller consists of output feedback and is suitable only for decentralized Single Input Single Output (SISO) systems.

However, the same strategy as used in this paper in order to develop the proposed simple MIMO LQ optimal PI controller will be used to formulate a simple Model Predictive Controller (MPC) with integral action in a upcoming paper. See e.g. Maciejowski (2002) for the MPC controller.

The rest of the paper is organized as follows. In Section 2 the optimal control problem is defined. In Section 3 the problem solution and the proposed LQ optimal controller with integral action for MIMO systems are presented. In Section 5 the optimal controller is compared with the conventional PI controller and the main differences and similarities are pointed out. In Section 6 the proposed LQ optimal controller with integral action is demonstrated on the problem of controlling some systems described with non-linear models, e.g. the quadruple tank process Johansson (2002) as well as three other examples. In Section 7 the proposed LQ optimal controller with integral action is illustrated in a practical experiment on the quadruple tank process. The first principles model is also compared with system identification based models. Some conclusions follow in Section 8. In Appendix A a MATLAB m-file script is provided for the easy application of the proposed method for LQ optimal controller with integral action.

\section{Problem formulation}

Given a process model

$$
\begin{aligned}
x_{k+1} & =A x_{k}+B u_{k}+v, \\
y_{k} & =D x_{k}+w
\end{aligned}
$$

where $x_{k} \in \mathbb{R}^{n}$ is the state vector, $u_{k} \in \mathbb{R}^{r}$ is the control input vector, $y_{k} \in \mathbb{R}^{m}$ is the output (measurement) vector, and $A, B$ and $D$ are known system matrices of appropriate dimensions. The disturbances $v$ and $w$ are both unknown, i.e., $v$ is an unknown constant or a slowly varying process disturbance, and $w$ is an unknown constant or a slowly varying measurement noise vector.

Note that the variables $u_{k}$ and $y_{k}$ in the model Eqs. (1) and (2) are the actual input and output variable, respectively. Furthermore, note that the model Eqs. (1) and (2) may arise from linearizing non-linear models around some nominal steady state and input variables, or from system identification based on trended variables. Hence, in these cases, the external noise variables $v$ and $w$ are known, but the resulting control algorithm to be presented in this paper is insensitive to these noise variables. Furthermore the system and the measurements may be influenced by drifts and in these cases the noise variables $v$ and $w$ may be unknown and slowly varying. Hence, the model Eqs. (1) and (2) is a realistic model.

We will study the LQ optimal controller when it is subjected to the following scalar performance index,

$$
\begin{gathered}
J_{i}=\frac{1}{2} x_{N}^{T} S x_{N} \\
+\frac{1}{2} \sum_{k=i}^{N-1}\left(\left(y_{k}-r\right)^{T} Q\left(y_{k}-r\right)+\Delta u_{k}^{T} P \Delta u_{k}\right),
\end{gathered}
$$

where $\Delta u_{k}=u_{k}-u_{k-1}$ is the control increment (deviation) and $r$ is a reference signal and $S, Q$ and $P$ are symmetric positive semi-definite weighting matrices of appropriate dimensions, $i$ is the initial time and often $i=0$ for simplicity of notation. The reference vector may be a time variant but for reasons of simplicity of the problem solution, we put $r_{k}=r$. The reference $r$ is treated as constant or slowly varying in the design phase of the LQ optimal controller with integral action for MIMO systems.

For large or infinite prediction horizons $N$ or when $S$ is chosen as the solution to the Riccati equation of the problem, then Eq. (3) is equivalent to using the index

$$
J_{i}=\frac{1}{2} \sum_{k=i}^{\infty}\left(\left(y_{k}-r\right)^{T} Q\left(y_{k}-r\right)+\Delta u_{k}^{T} P \Delta u_{k}\right)
$$




\section{Problem solution}

In order to solve the LQ optimal control problem we need a model which is independent of the unknown disturbances $v$ and $w$ in Eqs. (1) and (2). For the sake of generality we will focus on state space modeling.

From the state Equation (1) we have

$$
\Delta x_{k+1}=A \Delta x_{k}+B \Delta u_{k},
$$

where $\Delta x_{k}=x_{k}-x_{k-1}$. From the measurement equation (2) we have

$$
y_{k}=y_{k-1}+D \Delta x_{k} .
$$

Augmenting (5) with (6) gives the state space model

$$
\begin{gathered}
{\left[\begin{array}{l}
\Delta x_{k+1} \\
y_{k}
\end{array}\right]=\left[\begin{array}{ll}
A & 0_{n \times m} \\
D & I_{m \times m}
\end{array}\right]\left[\begin{array}{l}
\Delta x_{k} \\
y_{k-1}
\end{array}\right]+\left[\begin{array}{l}
B \\
0_{m \times r}
\end{array}\right] \Delta u_{k}} \\
y_{k}=\left[\begin{array}{ll}
D & I_{m \times m}
\end{array}\right]\left[\begin{array}{l}
\Delta x_{k} \\
y_{k-1}
\end{array}\right]
\end{gathered}
$$

and where as

Hence, the state space model given by Eqs. (12) and (13) with the performance index given by Eq. (14) define a standard LQ optimal control problem. We here assume $P>0$, the pair $(\tilde{A}, \tilde{B})$ is stabilizable and that the pair $(C, \tilde{A})$ is detectable where $C$ is the square root matrix of $\tilde{Q}$ such that $\tilde{Q}=C^{T} C$, in order for an optimal solution to exist.

The solution to the LQ optimal control problem, i.e. minimizing the performance index (14) with respect to the control deviation $\Delta u_{k}$ subject to the state Eq. (12), is given by the state feedback

$$
\Delta u_{k}=G \tilde{x}_{k}
$$

nd where the feedback matrix $G$ in eq. (15) is ob-

$$
G=-\left(P+\tilde{B}^{T} R \tilde{B}\right)^{-1} \tilde{B}^{T} R \tilde{A}
$$

The performance index (3) with $r=0$ and the state space model (7) and (8) define a standard LQ control problem. If $r$ is a non-zero constant reference then the measurements equation (8) can be written as

$$
y_{k}-r=y_{k-1}-r+D \Delta x_{k} .
$$

The state and output equations (7) and (8) can then be rewritten as

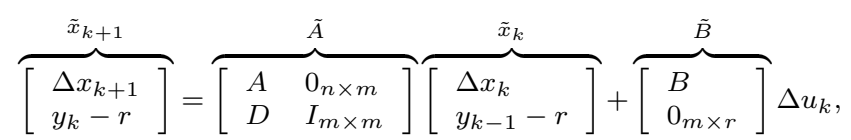

$$
\overbrace{y_{k}-r}^{\tilde{y}_{k}}=\overbrace{\left[\begin{array}{ll}
D & I_{m \times m}
\end{array}\right]}^{\tilde{D}} \overbrace{\left[\begin{array}{l}
\Delta x_{k} \\
y_{k-1}-r
\end{array}\right]}^{\tilde{x}_{k}} .
$$

The state space model (10) and (11) with the performance index (3) define a standard LQ optimal control problem.

Hence, we have a strictly proper state space model of the form

$$
\begin{aligned}
\tilde{x}_{k+1} & =\tilde{A} \tilde{x}_{k}+\tilde{B} \Delta u_{k}, \\
\tilde{y}_{k} & =\tilde{D} \tilde{x}_{k}
\end{aligned}
$$

Note that the index (4) yields

$$
\begin{aligned}
J_{i} & =\frac{1}{2} \sum_{k=i}^{\infty}\left(\tilde{y}_{k}^{T} Q \tilde{y}_{k}+\Delta u_{k}^{T} P \Delta u_{k}\right) \\
& =\frac{1}{2} \sum_{k=i}^{\infty}\left(\tilde{x}_{k}^{T} \tilde{Q} \tilde{x}_{k}+\Delta u_{k}^{T} P \Delta u_{k}\right),
\end{aligned}
$$

where the weighting matrix is $\tilde{Q}=\tilde{D}^{T} Q \tilde{D}$. where $R$ is the positive solution to the discrete time algebraic Riccati equation

$$
\begin{aligned}
R & =\tilde{Q}+\tilde{A}^{T} R \tilde{A}-\tilde{A}^{T} R \tilde{B}\left(P+\tilde{B}^{T} R \tilde{B}\right)^{-1} \tilde{B}^{T} R \tilde{A} \\
& =\tilde{Q}+G^{T} P G+(\tilde{A}+\tilde{B} G)^{T} R(\tilde{A}+\tilde{B} G),
\end{aligned}
$$

where the last formulation of the Riccati equation is known as the Joseph's stable version which ensures symmetry of the solution $R$. The solution to the LQ optimal control problem, Eqs.(16) and (17) is well known in the literature, see e.g. Anderson and Moore (1989) p. 53 or Lemma 11.2.1 in Söderström (1994) p. 291.

(10 Now from eq. (15) we find the following controller on incremental (velocity) form

$$
\Delta u_{k}=\overbrace{\left[\begin{array}{ll}
G_{1} & G_{2}
\end{array}\right]}^{G} \overbrace{\left[\begin{array}{l}
\Delta x_{k} \\
y_{k-1}-r
\end{array}\right]}^{\tilde{x}_{k}},
$$

which can be rewritten as $u_{k}=u_{k-1}+\Delta u_{k}$ or as

$$
u_{k}=u_{k-1}+G_{1} \Delta x_{k}+G_{2}\left(y_{k-1}-r_{k}\right) \text {, }
$$

where we are putting $r=r_{k}$ in eq. (18) to obtain the proposed controller eq. (19). The resulting controller eq. (19) has an appealing structure very similar to a PI controller on velocity form. See Sec. 5 for comparison.

Possible constraints are handled as with conventional PI controllers on velocity (incremental) form, e.g. as in Aström and Hägglund (1995) p.82. Notice also that it is simple to limit the rate of change $\Delta u_{k}$ of the control signal, and the control signal $u_{k}$, using the proposed LQ controller in eqs. (18) and (19).

A MATLAB m-file script for computing the LQ optimal feedback matrices $G_{1}$ and $G_{2}$ with the model matrices $A, B, D$ and the weighting matrices $Q$ and $P$ as arguments is provided in Appendix A. 
The weighting matrices $Q \geq 0$ and $P>0$ are usually chosen by some trial and error procedure for acceptable responses and performance. The weighting matrices may often be chosen as simple diagonal matrices, e.g. as $P=I$ and $Q=q I$ where $I$ is the identity matrix and $q>0$ a tuning parameter. See Sec. 6 for illustrating examples.

The LQ optimal controller (18) gives a zero steady state error, i.e. $y=r$ in steady state, since the closed loop system is stable owing to the properties of the LQ optimal controller (assuming the control variables are not saturated, i.e. the control variables are within allowed bounds).

Notice that it is possible to use $\tilde{Q} \in \mathbb{R}^{(n+m) \times(n+m)}$ in Eq. (14) directly as a weighting matrix in order to increase the degree of freedom in tuning the LQ optimal controller feedback matrices in Eq. (19). But owing to reasons of simplicity, we propose the strategy as presented.

\section{State observer for the state deviation}

The states are seldom measured in practice. In this case we can use a state observer or Kalman filter, Jazwinski (1989), Söderström (1994), to define the deviation state $\Delta x_{k}$. However, another solution is to define $\Delta x_{k}$ in terms of some past and known outputs $\ldots, y_{k-1}, y_{k}$ and some known inputs $\ldots, u_{k-1}$ and the model matrices $A, B$ and $D$.

A common solution to the problem of estimating the state, $x_{k}$, in a model of the form (1) and (2) in which the noise is colored, is to include a random walk (integrator) in order to estimate the non-zero part of the noise, $\bar{v}_{k}$ in addition to the state estimate $\bar{x}_{k}$. This is necessary in order for the innovations to become white. One can thereafter form the state deviation $\Delta \bar{x}_{k}=\bar{x}_{k}-\bar{x}_{k-1}$ which is needed in the control algorithm. However, another more simple solution in this situation is to design an observer for the deviation model (5) and (6). This gives a state observer for $\Delta \bar{x}_{k}$ of the form

$$
\Delta \bar{x}_{k+1}=A \Delta \bar{x}_{k}+B \Delta u_{k}+K\left(y_{k}-y_{k-1}-D \Delta \bar{x}_{k}\right),
$$

where the initial estimate $\Delta \bar{x}_{0}$ should be specified. A natural choice is $\Delta \bar{x}_{0}=0$.

System identification, e.g. the subspace system identification method DSR in Di Ruscio (2009) may also be used to directly identify the model matrices $A, B, D$ and the Kalman filter gain $K$ needed in the observer eq. (20). The DSR identified model matrices $A, B$ and $D$ may then also be used to develop the proposed
LQ controller eq. (19). This "model free" LQ optimal controller strategy is implemented on a practical laboratory process and illustrated with experimental results in Sec. 7.

Using a state observer in connection with the optimal controller eq. (19) leads to a Linear Quadratic Gausian (LQG) controller, (see e.g. Ch. 11 in Söderström (1994)). We are aware of the possible robustness problems with LQG controllers as demonstrated in, Doyle (1978). However, this possible problem is also involved in the common and widely used MPC controllers.

\section{Connection with the PI controller}

In this section we compare the structure of the proposed LQ controller eq. (19) with a PI controller on velocity (incremental) form.

A conventional PI controller can be written as

$u=K_{p} \frac{1+T_{i} s}{T_{i} s}(r-y)=K_{p}(r-y)+\frac{K_{p}}{T_{i}} \frac{1}{s}(r-y)$.

Defining the PI controller state $z$, as

$$
z=\frac{1}{s}(r-y) .
$$

Hence, the PI controller can in continuous time be written as

$$
\begin{aligned}
\dot{z} & =r-y, \\
u & =K_{p}(r-y)+\frac{K_{p}}{T_{i}} z .
\end{aligned}
$$

A discrete formulation of the PI controller is then

$$
\begin{aligned}
z_{k+1}-z_{k} & =h\left(r-y_{k}\right), \\
u_{k} & =K_{p}\left(r-y_{k}\right)+\frac{K_{p}}{T_{i}} z_{k},
\end{aligned}
$$

where $h$ is the sampling interval. A deviation formulation of the PI controller is then found as follows

$$
\begin{gathered}
u_{k}-u_{k-1} \\
=K_{p}\left(r-y_{k}\right)+\frac{K_{p}}{T_{i}} z_{k}-\left(K_{p}\left(r-y_{k-1}\right)+\frac{K_{p}}{T_{i}} z_{k-1}\right) \\
=-K_{p}\left(y_{k}-y_{k-1}\right)+\frac{K_{p}}{T_{i}}\left(z_{k}-z_{k-1}\right) .
\end{gathered}
$$

From (25) we have that $z_{k}-z_{k-1}=h\left(r-y_{k-1}\right)$. Substituting this into (27) gives

$$
u_{k}=u_{k-1}+G_{1}\left(y_{k}-y_{k-1}\right)+G_{2}\left(y_{k-1}-r\right) .
$$

where

$$
G_{1}=-K_{p}, G_{2}=-\frac{K_{p}}{T_{i}} h
$$


Furthermore, using that $y_{k}=D x_{k}+w$ gives

$$
u_{k}=u_{k-1}+G_{1} D \Delta x_{k}+G_{2}\left(y_{k-1}-r\right) .
$$

The above discussion shows that the PI controller is exactly of the same structure as the LQ optimal controller (19). The difference is that the optimal controller takes feedback from the deviation state vector $\Delta x_{k}=x_{k}-x_{k-1}$ while the PI-controller only uses feedback from the output deviation $\Delta y_{k}=D \Delta x_{k}$.

\section{Numerical examples}

\section{Example 6.1 (Quadruple tank process)}

Consider the quadruple tank process, Johansson (2002), with the non-linear state space model derived from mass balances and Bernoulli's/Torricelli's law. By equating the potential energy and kinetic energy, i.e. $m g h=\frac{1}{2} m v^{2}$ and solving for the velocity we obtain $v=\sqrt{2 g h}$. Multiplying with the area, a, of the outlet hole of the tank we obtain the volumetric flowrate, $q$, out of the tank as $q=a v=a \sqrt{2 g h}$.

Hence, a mass balance of the four tank process gives the state space model

$$
\begin{aligned}
& A_{1} \dot{x}_{1}=-a_{1} \sqrt{2 g x_{1}}+a_{3} \sqrt{2 g x_{3}}+\gamma_{1} k_{1} u_{1}, \\
& A_{2} \dot{x}_{2}=-a_{2} \sqrt{2 g x_{2}}+a_{4} \sqrt{2 g x_{4}}+\gamma_{2} k_{2} u_{2}, \\
& A_{3} \dot{x}_{3}=-a_{3} \sqrt{2 g x_{3}}+\left(1-\gamma_{2}\right) k_{2} u_{2}, \\
& A_{4} \dot{x}_{4}=-a_{4} \sqrt{2 g x_{4}}+\left(1-\gamma_{1}\right) k_{1} u_{1},
\end{aligned}
$$

where $A_{i} \forall i=1, \ldots, 4$ is the cross-section area of tank $i, a_{i} \forall i=1, \ldots, 4$ is the cross-section area of the outlet pipe of tank $i$.

The flow $k_{1} u_{1}$ from pump 1 may be divided into a flow $\gamma_{1} k_{1} u_{1}$ into tank 1 and a flow $\left(1-\gamma_{1}\right) k_{1} u_{1}$ to tank 4, i.e. such that the flow from pump number 1 is $k_{1} u_{1}=\gamma_{1} k_{1} u_{1}+\left(1-\gamma_{1}\right) k_{1} u_{1}$. Similarly, the flow $k_{2} u_{2}$ from the second pump may be divided into a flow $\gamma_{2} k_{2} u_{2}$ into tank 2 and a flow $\left(1-\gamma_{2}\right) k_{2} u_{2}$ into tank 3. Here $\gamma_{1}$ and $\gamma_{2}$ are fixed parameters. The system is non-minimum phase when choosing these parameters such that, $0<\gamma_{1}+\gamma_{2}<1$, and the system is minimum phase when, $1<\gamma_{1}+\gamma_{2}<2$. The numerical values for the above parameters, as well as nominal values for the states and control inputs, are chosen as presented in Johansson (2002).

The 4 tank process is studied in a number of papers, see e.g. Gatzke et al. (2000) where Internal Model Control (IMC) and Dynamic Matrix Control (DMC) were used to control the 4 tank process. Here we use the proposed LQ optimal controller with integral action as presented in Sec. 3.

The results after using the $L Q$ optimal controller, eq. (19), in order to control the non-linear model eqs. (31)(34) are presented in Figures 1 and 2. The weighting matrices were chosen simply as $P=I_{2}$ and $Q=I_{2}$ for both the minimum and non-minimum phase cases.
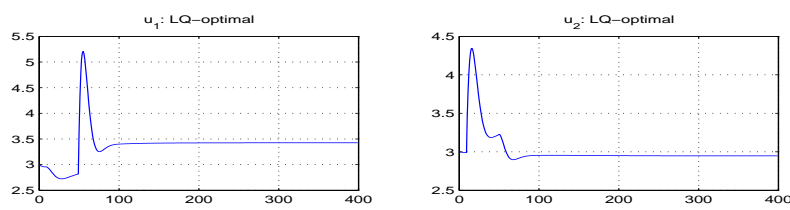

$y_{1}$ : Level tank 1
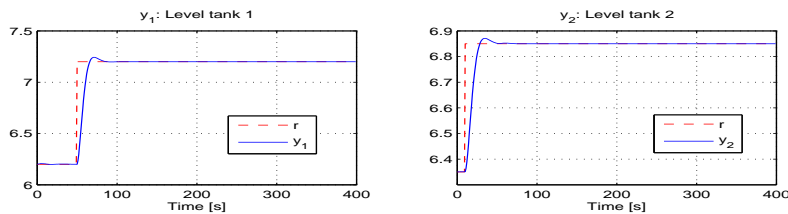

Figure 1: Simulation of the quadruple tank process and the minimum phase case in Example 6.1 with LQ optimal control with integral action.
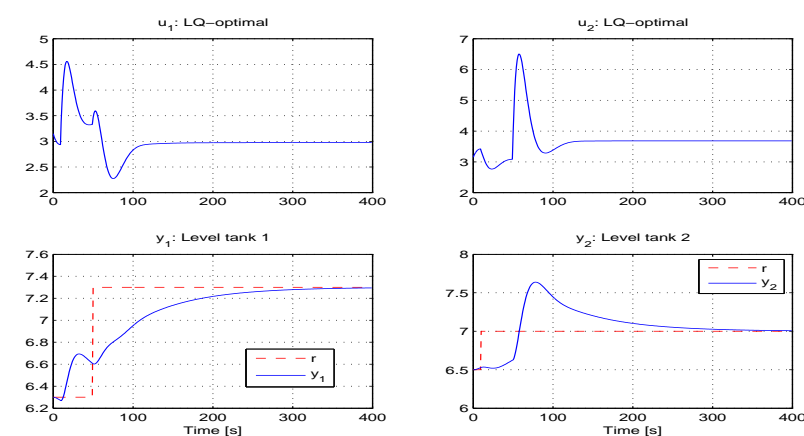

Figure 2: Simulation of the quadruple tank process and the non-minimum phase case in Example 6.1 with LQ optimal control with integral action.

\section{Example 6.2 (Isothermal chemical reactor)}

$A$ chemical isothermal reactor with a reaction $s A \rightarrow^{k} B$ , which can be modeled as

$$
\begin{aligned}
& \dot{x}_{1}=\frac{u_{1}}{V}\left(u_{2}-x_{1}\right)-s k x_{1}^{2}, \\
& \dot{x}_{2}=-\frac{u_{1}}{V} x_{2}+k x_{1}^{2},
\end{aligned}
$$

where $V=1, k=1$ and $s=2 . u_{1}$ is the flow rate, $u_{2}$ the feed concentration, $V$ the volume and $k$ a reaction velocity constant. The states $x_{1}$ and $x_{2}$ are the molar compositions of the substances $A$ and $B$ respectively.

The steady state control variables $u_{1}^{s}=10$ and $u_{2}^{s}=$ 1 give the steady states $x_{1}^{s}=0.8541$ and $x_{2}^{s}=0.0729$. Linearizing around the steady state gives the linear model

$$
\Delta \dot{x}=A_{c} \Delta x+B_{c} \Delta u
$$


where $\Delta x=x-x^{s}$ and $\Delta u=u-u^{s}$ and

$$
\begin{aligned}
& A_{c}=\left[\begin{array}{ll}
\frac{\partial f_{1}}{\partial x_{1}} & \frac{\partial f_{1}}{\partial x_{2}} \\
\frac{\partial f_{2}}{\partial x_{1}} & \frac{\partial f_{2}}{\partial x_{2}}
\end{array}\right]_{x^{s}, u^{s}}
\end{aligned}
$$

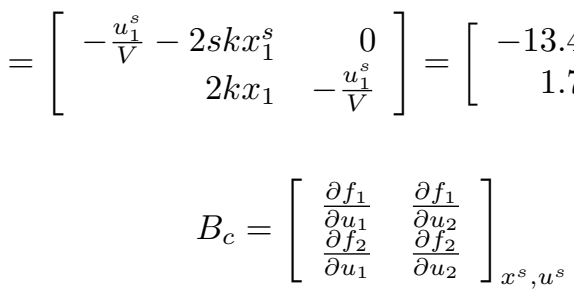

$$
\begin{aligned}
& =\left[\begin{array}{rr}
\frac{u_{2}^{s}}{V_{s}} & \frac{u_{1}^{s}}{V} \\
-\frac{x_{2}^{s}}{V} & 0
\end{array}\right]=\left[\begin{array}{rr}
1.0 & 10.0 \\
-0.0729 & 0
\end{array}\right] \text {. }
\end{aligned}
$$
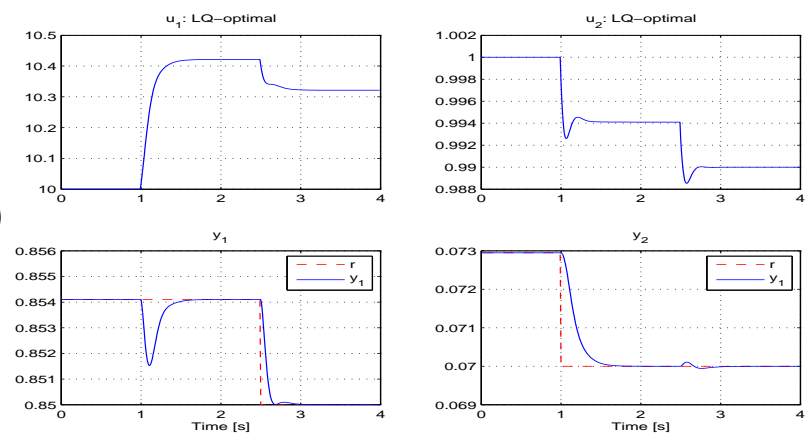

A discrete time model is obtained by using a zero order hold on the input and a sampling interval $h=0.01$, i.e.,

$$
\begin{aligned}
x_{k+1} & =A x_{k}+B u_{k}+v \\
y_{k} & =D x_{k}
\end{aligned}
$$

where

$$
\begin{gathered}
A=e^{A_{c} h}=\left[\begin{array}{rr}
0.8744 & 0 \\
0.0152 & 0.9048
\end{array}\right], \\
B=A_{c}^{-1}\left(e^{A_{c} h}-I\right) B_{c}=\left[\begin{array}{rr}
0.0094 & 0.0936 \\
-0.0006 & 0.0008
\end{array}\right], \\
D=\left[\begin{array}{ll}
1 & 0 \\
0 & 1
\end{array}\right], v=x^{s}-A x^{s}-B u^{s} .
\end{gathered}
$$

Choosing a $L Q$ criterion

$$
J_{i}=\frac{1}{2} \sum_{k=i}^{N}\left(\left(y_{k}-r\right)^{T} Q\left(y_{k}-r\right)+\Delta u_{k}^{T} P \Delta u_{k}\right),
$$

with

$$
P=\left[\begin{array}{rr}
1 & 0 \\
0 & 100
\end{array}\right], Q=\left[\begin{array}{rr}
50 & 0 \\
0 & 100
\end{array}\right],
$$

gives the $L Q$ optimal control

$$
u_{k}=u_{k-1}+G_{1} \Delta x_{k}+G_{2}\left(y_{k-1}-r\right),
$$

where

$$
\begin{aligned}
& G_{1}=\left[\begin{array}{rr}
-15.7253 & 55.7233 \\
-1.9714 & -6.5884
\end{array}\right], \\
& G_{2}=\left[\begin{array}{rr}
-4.7639 & 6.2149 \\
-0.3639 & -0.7540
\end{array}\right] .
\end{aligned}
$$

Simulation results after changes in the reference signal $r$ are illustrated in Figure 3.
Figure 3: Simulation of the chemical reactor in Example 6.2 with LQ-optimal control.

\section{Example 6.3 (Van de Vusse chemical reactor)}

A chemical isothermal reactor (Van de Vusse) is studied in this example. The relationship from the feed flow rate $u$ into the reactor to the concentration of the product $y$ at the outlet of the reactor is modeled by the following non-linear state space model.

$$
\begin{aligned}
\dot{x_{1}} & =-k_{1} x_{1}-k_{3} x_{1}^{2}+\left(v-x_{1}\right) u \\
\dot{x_{2}} & =k_{1} x_{1}-k_{2} x_{2}-x_{2} u \\
y & =x_{2}
\end{aligned}
$$

where the reaction rate coefficients are given by $k_{1}=$ $50, k_{2}=100, k_{3}=10$. The concentration of the byproduct into the reactor, $v$, is treated as an unknown constant or slowly varying disturbance with nominal value $v^{s}=10$. Choosing a steady state control $u^{s}=25$ gives the steady states $x_{1}^{s}=2.5$ and $y^{s}=x_{2}^{s}=1$.

A linearized model around the steady state is given by

$$
\Delta \dot{x}=A_{c} \Delta x+B_{c} \Delta u+C_{c} \Delta v
$$

where $\Delta x=x-x^{s}, \Delta u=u-u^{s}$ and $\Delta v=v-v^{s}$, and

$$
\begin{gathered}
A_{c}=\left[\begin{array}{ll}
\frac{\partial f_{1}}{\partial x_{1}} & \frac{\partial f_{1}}{\partial x_{2}} \\
\frac{\partial f_{2}}{\partial x_{1}} & \frac{\partial f_{2}}{\partial x_{2}}
\end{array}\right]_{x^{s}, u^{s}} \\
=\left[\begin{array}{rr}
-k_{1}-2 k_{3} x_{1}^{s}-u^{s} & 0 \\
k_{1} & -k_{2}-u
\end{array}\right]=\left[\begin{array}{rr}
-125 & 0 \\
50 & -125
\end{array}\right](54) \\
B_{c}=\left[\begin{array}{c}
\frac{\partial f_{1}}{\partial u} \\
\frac{\partial f_{2}}{\partial u}
\end{array}\right]_{x^{s}, v^{s}} \\
=\left[\begin{array}{r}
v^{s}-x_{1}^{s} \\
-x_{2}^{s}
\end{array}\right]=\left[\begin{array}{l}
7.5 \\
-1
\end{array}\right] .
\end{gathered}
$$

Notice that $C_{c}$ is computed similar as $B_{c}$ but is not needed. A discrete time model is obtained by using a 
zero order hold on the input and a sampling interval $h=0.002$, i.e.,

$$
\begin{aligned}
x_{k+1} & =A x_{k}+B u_{k}+v, \\
y_{k} & =D x_{k}
\end{aligned}
$$

where

$$
\begin{gathered}
A=e^{A_{c} h}=\left[\begin{array}{rr}
0.7788 & 0 \\
0.0779 & 0.7788
\end{array}\right] \\
B=A_{c}^{-1}\left(e^{A_{c} h}-I\right) B_{c}=\left[\begin{array}{r}
0.0133 \\
-0.0011
\end{array}\right] \\
D=\left[\begin{array}{ll}
0 & 1
\end{array}\right], v:=x^{s}-A x^{s}-B u^{s}+C\left(v-v^{s}\right)
\end{gathered}
$$

Notice that $C$ is computed similar as $B$ but is not needed because the $L Q$ optimal controller is independent of the constant disturbance $v$ in the state Eq. (56) (assuming a constant or slowly varying disturbance in the reactor). Choosing a $L Q$ criterion

$$
J_{i}=\frac{1}{2} \sum_{k=i}^{\infty}\left(Q\left(y_{k}-r\right)^{2}+P \Delta u_{k}^{2}\right)
$$

with

$$
P=1, Q=500
$$

gives the $L Q$ optimal control

$$
u_{k}=u_{k-1}+G_{1} \Delta x_{k}+G_{2}\left(y_{k-1}-r\right),
$$

where

$$
G_{1}=\left[\begin{array}{ll}
-23.4261 & -84.5791
\end{array}\right], G_{2}=-20.0581 .
$$

Simulation results after changes in the reference signal $r$ are illustrated in Figure 4 .
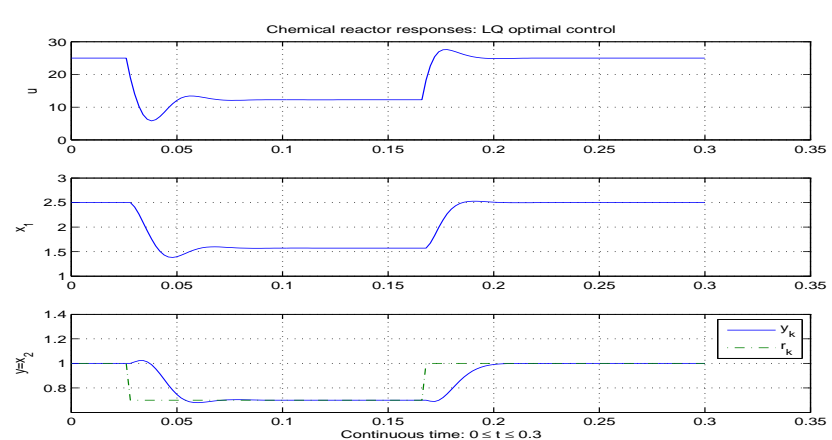

Figure 4: Simulation of the chemical reactor in Example 6.3 with LQ-optimal control.

\section{Example 6.4 (Distillation column)}

One advantage of the presented $L Q$ optimal control is that the control is designed in discrete time. Continuous processes with slow dominant dynamics are often controlled with a digital/discrete controller. If the sampling time is large then a continuous time controller design may give poor results when used as a discrete controller. We will here illustrate the simple discrete time $L Q$ optimal control design for a distillation column.

Consider a distillation column with eight trays and a relative volatility $\alpha=2.993$. Let the control variable $u_{1}=R$ be the reflux to the column and $u_{2}=V$ be the flow rate of vapor in the column. The composition of the top product $x_{8}=x_{D}$ and the composition of the bottom product $x_{1}=x_{B}$ are treated as measured output variables. The feed flow rate $F$ and the composition $x_{F}$ of the light product in $F$ are both treated as unknown constant or slowly varying disturbances.

The continuous non-linear model with $n=8$ states is first linearized around the steady state operating point $R^{s}=2, V^{s}=2.5, F^{s}=1$ and $x_{F}^{s}=0.5$. This gives a continuous time linear model of the form

$$
\begin{aligned}
\dot{\Delta x} & =A_{c} \Delta x+B_{c} \Delta u+C_{c} \Delta v \\
\Delta y & =D \Delta x .
\end{aligned}
$$

This model is then discretized with a sample interval of $h=5[\mathrm{~min}]$. This gives a discrete time model of the form

$$
\begin{aligned}
x_{k+1} & =A x_{k}+B u_{k}+v, \\
y_{k} & =D x_{k}+w .
\end{aligned}
$$

Choosing a $L Q$ criterion

$$
J_{i}=\frac{1}{2} \sum_{k=i}^{N}\left(\left(y_{k}-r\right)^{T} Q\left(y_{k}-r\right)+\Delta u_{k}^{T} P \Delta u_{k}\right),
$$

with

$$
P=\left[\begin{array}{ll}
1 & 0 \\
0 & 1
\end{array}\right], Q=2500\left[\begin{array}{ll}
1 & 0 \\
0 & 1
\end{array}\right]
$$

gives the $L Q$ optimal control

$$
u_{k}=u_{k-1}+G_{1} \Delta x_{k}+G_{2}\left(y_{k-1}-r\right) \text {, }
$$

where

$$
\begin{gathered}
G_{1}=\left[\begin{array}{llll}
12.8099 & 0.9303 & 0.3961 & -0.3187 \\
30.3424 & 2.2003 & 1.4407 & 0.5590 \\
-1.5158 & -3.2992 & -6.3394 & -50.2082 \\
-0.6629 & -2.0994 & -4.1887 & -30.6734
\end{array}\right]
\end{gathered}
$$




$$
G_{2}=\left[\begin{array}{ll}
10.0833 & -29.5242 \\
24.6664 & -17.1829
\end{array}\right] .
$$

The linear controller (71)-(73) on the deviation form is used in this example to control the non-linear distillation column model with eight states. If the state vector is not available, then we may use a state observer or compute an expression for $\Delta x_{k}$ from some past inputs and outputs, e.g. as in the Extended Model Predictive Control (EMPC) algorithm, Di Ruscio and Foss (1998). Simulation results after changes in the reference signal $r$ are illustrated in Figure 5.
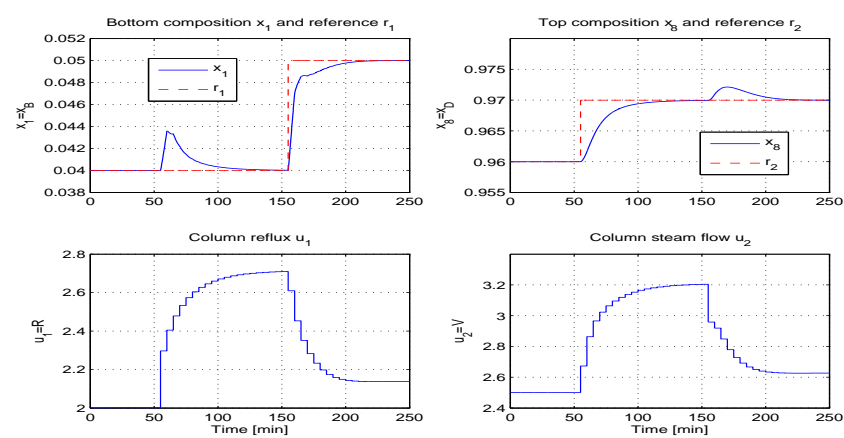

Figure 5: Simulation of the distillation column in Example 6.4 with LQ optimal control.

\section{Experimental results on a quadruple tank process}

The results from practical experiments on a quadruple tank laboratory process will be presented in this section.

The sampling rate in all experiments is one second. We started with empty tanks in all experiments. Hence, this may be viewed as a test for robustness for unknown non-linearities when using the proposed LQ controller. The quadruple tank process setup results in a non-minimum phase behavior.

The experiments are described in the following items.

1. An open loop input experiment is designed as illustrated in Fig (6) and the corresponding outputs, i.e. the levels in the two lower tanks, also illustrated in Fig (7).

2. The input and output data are collected into data matrices $U \in \mathbb{R}^{N \times 2}$, and $Y \in \mathbb{R}^{N \times 2}$ where the number of samples is $N=5459$. The first
$N_{\text {ID }}=4000$ first samples were used for identification. Hence, the last 1459 samples may be used for validation of the identified state space models. The data were also centered before use in the identification methods.

3. A First Principles (FP) model, very similar to the one presented in Example 6.1, were fitted to the process as well as believed possible. Using the input experiment as illustrated in Fig. (6) gave the simulated outputs as illustrated in Fig. (7). The Prediction Error (PE) criterion evaluated for the validation data was $V_{\mathrm{fp}}=7.57$.

4. The MATLAB IDENT Toolbox system identification function pem.m where used to identify a $n=4$ order state space model. The simulated outputs are illustrated in Fig. (7). The $\mathrm{PE}$ criterion evaluated for the validation data was $V_{\mathrm{PEM}}=3.38$.

5. The subspace system identification method, Di Ruscio (2009) was used. The best DSR model with $n=4$ states were found with parameters $L=2$ and $J=29$. The simulated outputs are illustrated in Fig. (7). The PE criterion evaluated for the validation data was $V_{\mathrm{DSR}}=3.07$.

6. Two SISO PI controllers were tuned by using the model based tuning method in Di Ruscio (2010). The model used was the DSR model. The experimental results using this decentralized control strategy are illustrated in Figs. (8) and (9).

7. The LQ optimal control strategy eq. (19) was implemented. The Kalman filter identified by the DSR method was used to identify the present state deviation $\Delta x_{k}=x_{k}-x_{k-1}$ needed in the controller. The experimental results using this LQ optimal controller with integral action strategy are illustrated in Figs. (8) and (9).

The conclusions drawn from this experimental results are discussed in the following.

Interestingly the identified state space models, both from PEM and DSR, fit the real data better than the FP model. Here the simulated output, i.e. the behavior from the input $\mathrm{u}$, to the output $\mathrm{y}$, is used in order to calculate the $\mathrm{PE}$ criterion. The results using the FP model, the PEM model and the DSR model are $V_{\mathrm{fp}}=7.57, V_{\mathrm{PEM}}=3.38$ and $V_{\mathrm{DSR}}=3.07$, respectively. Interestingly the DSR model fit the validation data slightly better than the PEM model.

Based on this conclusion we are using the identified DSR model for both tuning the PI controllers and for use in the LQ optimal controller with integral action strategy eq. (19). The deterministic part of the 
model, i.e. $x_{k+1}=A x_{k}+B u_{k}$ and $y_{k}=D x_{k}$, was used to tune the PI controller strategy (by first using the RGA pairing strategy, Bristol (1966), Skogestad and Postlethwaite (1996)), as well as for the calculation of the feedback matrices $G_{1}$ and $G_{2}$. Furthermore the DSR identified Kalman filter gain matrix $K$ was used in the Kalman filter on deviation form eq. (20), for estimating the deviation states $\Delta x_{k}$ needed in eq. (19).

As we see from Figs. (8) and (9) the LQ strategy works very well compared to the PI controller strategy. This is justified by comparing the Integrated Absolute (IAE) indices. The DSR model with the LQ optimal controller in Eq. (19) gave IAE indices 1.6849 and 1.3290 for level one and two, respectively, and for the PI controllers 2.2723 and 2.5141 for level one and two, respectively. It is also worth mentioning that it is very difficult to tune PI controllers for this process due to the non-minimum phase behavior of the process.
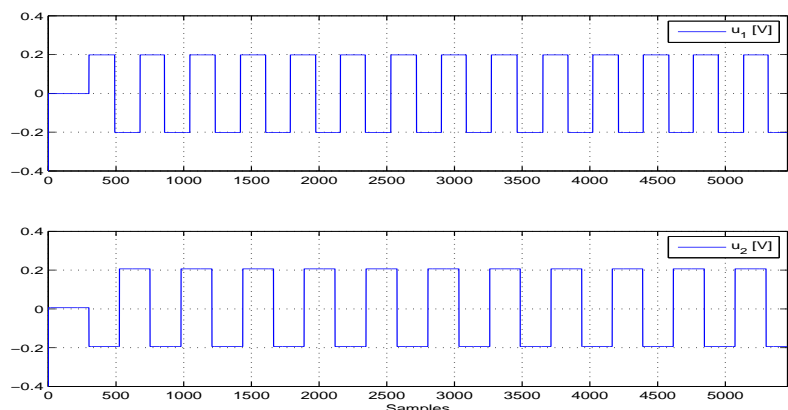

Figure 6: Open loop system identification input experiment, i.e. the volt input to the pumps.

\section{Concluding remarks}

A simple LQ optimal controller with integral action on velocity (incremental) form for MIMO systems is proposed. The proposed LQ controller is demonstrated to work well on four simulation examples. Furthermore, practical experiments show that the proposed LQ controller works well on a quadruple tank laboratory process.

\section{Acknowledgment}

The author acknowledges Mr Danuskha Dodampe Gamag who did most of the practical experiments in Sec. 7 of this paper.

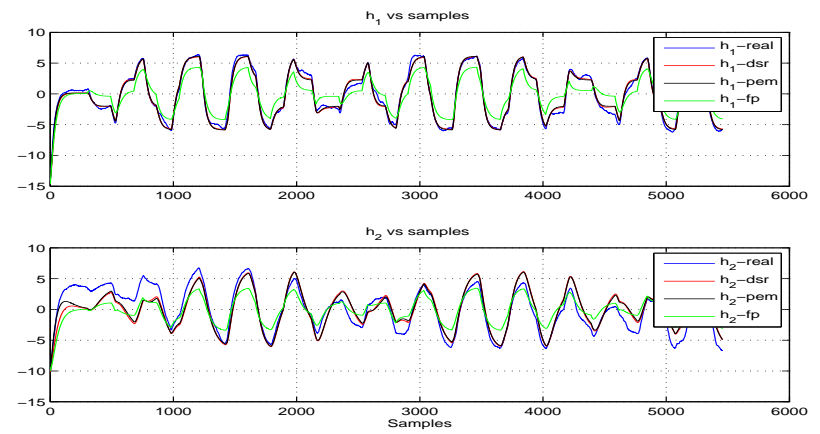

Figure 7: This figure illustrates the real measurements of the level in the two lower tanks as well as the corresponding simulated outputs of the system identification models, from DSR and PEM, as well as the simulated outputs from the first principles model.

\section{A. MATLAB script for computation}

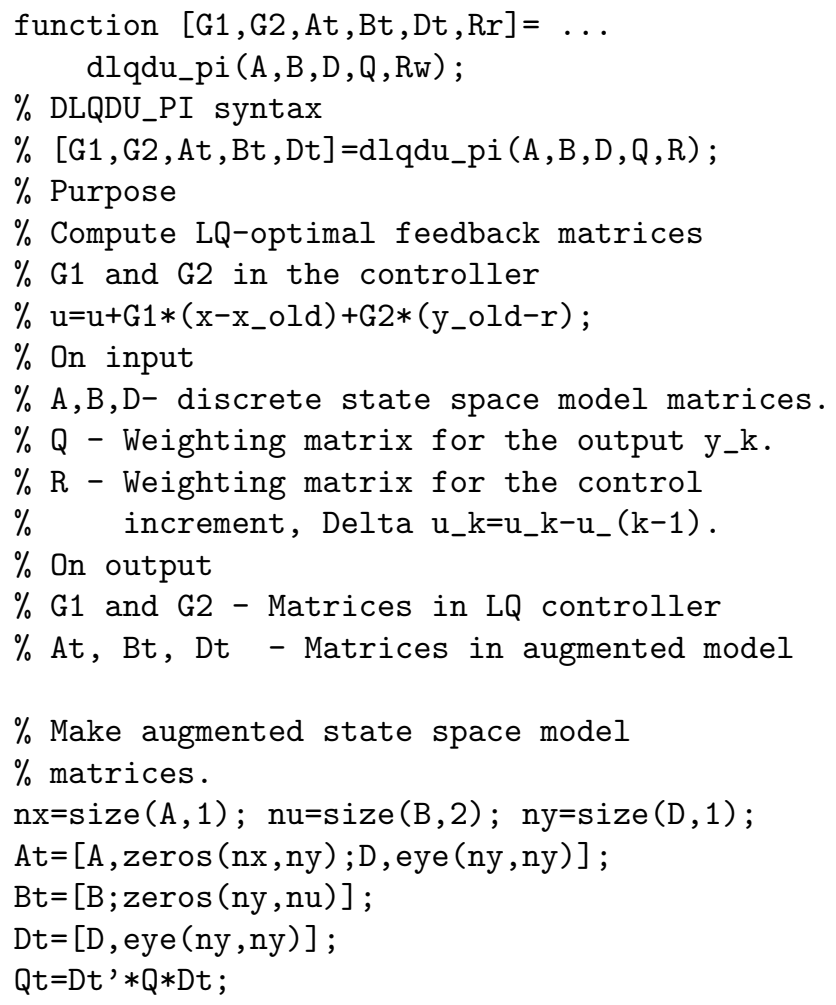




\section{References}

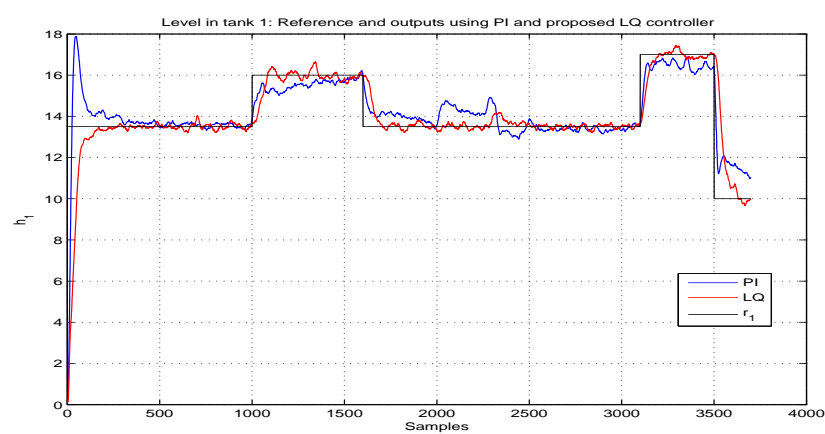

Figure 8: Quadruple tank process. Level in tank one. Illustrating the reference and the outputs from the process controlled by two single loop PI controllers, and the proposed LQ optimal controller with integral action. The LQ controller was constructed by using the DSR method for system identification. The DSR model was used to identify a Kalman filter for the system. The states were estimated with this Kalman filter and the deterministic part of the model was used to design the controller.

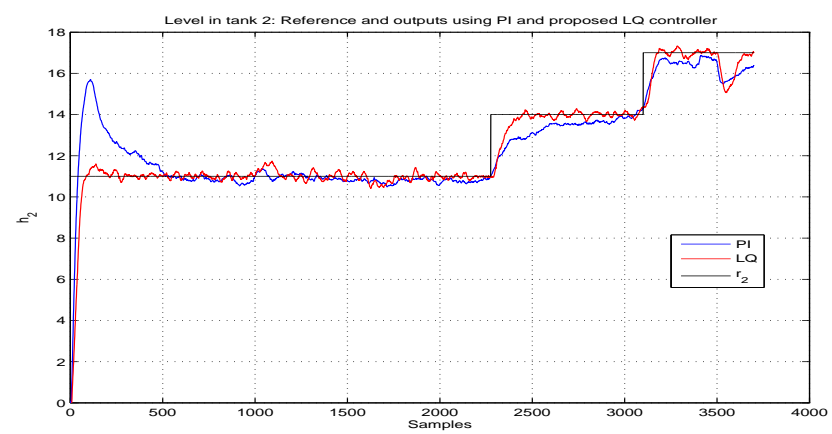

Figure 9: Quadruple tank process. Level in tank two. Illustrating the reference and the outputs from the process controlled by two single loop PI controllers, and the proposed LQ optimal controller with integral action. The LQ controller where constructed by using the DSR method for system identification. The DSR model was used to identify a Kalman filter for the system. The states were estimated with this Kalman filter and the deterministic part of the model was used to design the controller.
Anderson, B. D. O. and Moore, J. B. Optimal Control: Linear Quadratic Methods. Prentice-Hall International Editions, 1989.

Åström, K. and Hägglund, T. PID Controllers: Theory, Design, and Tuning. Instrument Society of America, 1995.

Bristol, E. H. On a new measure of interactions for multivariable process control. Transactions on Automatic Control, 1966. 11(11):113-114. doi:10.1109/TAC.1966.1098266.

Di Ruscio, D. Closed and Open Loop Subspace System Identification of the Kalman Filter. Modeling, Identification and Control, 2009. 30(2):71-86. doi:10.4173/mic.2009.2.3.

Di Ruscio, D. On Tuning PI Controllers for Integrating Plus Time Delay Systems. Modeling, Identification and Control, 2010. 31(4):145-164. doi:10.4173/mic.2010.4.3.

Di Ruscio, D. and Foss, B. On model based predictive control. In The 5th IFAC Symposium on Dynamics and Control of Process Systems. 1998.

Doyle, J. C. Guaranteed Margins for LQG Regulators. IEEE Transactions on Automatic Control, 1978. 23(4):756-757. doi:10.1109/TAC.1978.1101812.

Gatzke, E. P., Meadows, E. S., Wang, C., and Doyle, F. J. I. Model based control of a four-tank system. Computers and Chemical Engineering, 2000. 24(2):1503-1509. doi:10.1016/S0098-1354(00)00555$\mathrm{X}$.

Jazwinski, A. H. Stochastic processes and filtering theory. Academic Press, 1989.

Johansson, K. H. Interaction bounds in multivariable control systems. Automatica, 2002. 38(2):1045-1051. doi:10.1016/S0005-1098(01)00285-0.

Kwakernaak, H. and Sivan, R. Linear Optimal Control Systems. Wiley-Interscience, 1972.

Maciejowski, J. Predictive control: with constraints. Pearson Education, 2002.

Skogestad, S. and Postlethwaite, I. Multivariable Feedback Control: Analysis and Design. Wiley, 1996.

Söderström, T. Discrete-time Stochastic Systems: Estimation and Control. Prentice Hall, 1994. 